\title{
pqqA is not required for biosynthesis of pyrroloquinoline quinone in Methylobacterium extorquens AM1
}

\author{
Hirohide Toyama† and Mary E. Lidstrom
}

Department of Chemical Engineering, University of Washington, Box 351750 , Seattle, WA 98195, USA

\author{
Author for correspondence: Mary E. Lidstrom. Tel: +1 206616 5282. Fax: +1 2066165721. \\ e-mail: lidstrom@u.washington.edu
}

\begin{abstract}
Methylobacterium extorquens AM1 is a facultative methylotroph that oxidizes methanol via the pyrroloquinoline quinone (PQQ)-linked enzyme methanol dehydrogenase. In $M$. extorquens AM1 and other PQQ-synthesizing bacteria, several genes are involved in the synthesis of $P Q Q$ and one of these, $P q q A$, has been proposed to encode a peptide precursor of PQQ. In other PQQsynthesizing bacteria, PqqA is required for PQQ production. In this study, it is shown that both deletion and insertion mutants of pqqA in $M$. extorquens AM1 grow normally on methanol and produce PQQ. The level of PQQ production is reduced in the insertion mutant, but it is sufficient to allow normal growth on methanol. These results suggest either that a different peptide in $M$. extorquens AM1 can substitute for PqqA in pqqA mutants, or that PqqA-like peptides may not be obligatory precursors of PQQ. In addition, it is shown that the methanol oxidation transcriptional regulator gene, $\mathbf{m x b M}$, is required for normal methanol induction of PQQ synthesis.
\end{abstract}

Keywords: pyrroloquinoline quinone, PQQ, methylotrophs, $p q q A$

\section{INTRODUCTION}

Pyrroloquinoline quinone, PQQ, is the cofactor of methanol dehydrogenase (MDH), which is the enzyme that oxidizes methanol to formaldehyde in Gramnegative methylotrophic bacteria (Anthony et al., 1994). Several enzymes besides MDH found in non-methylotrophic bacteria also contain PQQ (Matsushita \& Adachi, 1993). The genes involved in biosynthesis of PQQ have been cloned and sequenced from Acinetobacter calcoaceticus (Goosen et al., 1989), Klebsiella pneumoniae (Meulenberg et al., 1992), Pseudomonas fluorescens (Schnider et al., 1995) and Methylobacterium extorquens AM1 (Morris et al., 1994; Springer et al., 1996; Toyama et al., 1997). In K. pneumoniae, the genes are termed pqqABCDEF (Meulenberg et al., 1992). Tyrosine and glutamate have been shown to be the precursors of PQQ synthesis (Kleef \& Duine, 1988; Houck et al., 1991), but the pathway is still unknown.

An open reading frame encoding a short peptide (22-29 amino acids) is found at the start of the known $p q q$ gene

†Present address: Department of Biological Chemistry, Yamaguchi University, Yamaguchi 753, Japan.

Abbreviations: $\mathrm{MDH}$, methanol dehydrogenase; PQQ, pyrroloquinoline quinone. clusters (Goosen et al., 1989; Meulenberg et al., 1992; Morris et al., 1994; Schnider et al., 1995; Gomelsky et al., 1996). The gene encoding this peptide is required for PQQ synthesis in A. calcoaceticus (Goosen et al., 1992), in Escherichia coli containing the K. pneumoniae pqq genes (Meulenberg et al., 1992), and in K. pneumoniae (Velterop et al., 1995). The deduced amino acid sequences of these peptides showed some similarity and included conserved tyrosine and glutamate residues (Goosen et al., 1989; Meulenberg et al., 1992; Morris et al., 1994; Schnider et al., 1995; Gomelsky et al., 1996). In addition, another gene required for PQQ synthesis, $p q q F$, shows similarity to a family of proteases that might be involved in processing the peptide (Meulenberg et al., 1992). Therefore, it has been proposed that the peptide might be the precursor of PQQ synthesis (Goosen et al., 1992; Meulenberg et al., 1992).

In Methylobacterium extorquens AM1, the genes for PQQ synthesis are found in two clusters, $p q q A B(C / D) E$ and pqqFG (Toyama et al., 1997). These gene designations standardize the nomenclature with that of $K$. pneumoniae. These genes in Methylobacterium strains were formerly called pqqDGCBA (Morris et al., 1994) and $p q q E F$ (Springer et al., 1996), respectively. In $M$. extorquens AM1, $p q q C$ and $p q q D$ are not separate genes. Instead, they are fused into a single gene, $p q q C / D$ 
Table 1. Bacterial strains and plasmids

\begin{tabular}{|c|c|c|}
\hline Strain or plasmid & Relevant trait(s) & Source or reference \\
\hline \multicolumn{3}{|l|}{ E. coli } \\
\hline $\mathrm{DH} 5 \alpha$ & $\mathrm{r}^{-} \mathrm{m}^{+}$recA1 lacZYA $\phi 80 \mathrm{~d} l a c \Delta(\operatorname{lac} Z) \mathrm{M} 15$ & $\begin{array}{l}\text { Bethesda Research } \\
\text { Laboratories }\end{array}$ \\
\hline S17-1 & $\begin{array}{l}\text { recA oriT integrated RP4-2-Tc: :Mu- } \\
\mathrm{Km}:: \operatorname{Tn} 7\end{array}$ & Simon et al. (1983) \\
\hline GM2163 & $\mathrm{F}^{-}$dam-13:: Tn9 $\left(\mathrm{Cam}^{\mathrm{r}}\right) d c m-6 m c r A$ & New England Biolabs \\
\hline \multicolumn{3}{|l|}{ M. extorquens } \\
\hline AM1 rif & Wild-type & Nunn \& Lidstrom (1986) \\
\hline UV45 & $p q q E$ & Nunn \& Lidstrom (1986) \\
\hline EMS12 & $p q q C$ & Morris et al. (1994) \\
\hline UV46 & $p q q B$ & Nunn \& Lidstrom (1986) \\
\hline $3 b$ & $\Delta[m x b M p q q A B(C / D) E]$ & This study \\
\hline D2 & $p q q A:: \mathrm{Km}$ & This study \\
\hline \multicolumn{3}{|l|}{ Plasmids } \\
\hline pRK310 & $\mathrm{Tc}^{\mathrm{r}}$ IncP1, broad-host-range vector & Ditta et al. (1985) \\
\hline pUC18/19 & $A^{r} l a c Z^{\prime}$ & Yanisch-Perron et al. (1985) \\
\hline pAYC61 & $A p^{r} T^{r}$ ori $T$, suicide vector & Chistoserdov et al. (1994) \\
\hline $\mathrm{pALS} 519 \mathrm{~b} / \mathrm{f}$ & $\begin{array}{l}\text { pUC19 containing } 10 \mathrm{~kb} \text { Pst I fragment } \\
{[m x b D M \text { and } p q q A B(C / D) E]}\end{array}$ & This study \\
\hline $\mathrm{pALS5Kan61b/f}$ & $\begin{array}{l}\text { pALS519b/f:: Km }{ }^{\mathrm{r}}(\text { BglII site }) \Delta 4 \cdot 1 \mathrm{~kb} B g l \mathrm{lI} \\
\text { fragment }(m x b D \Delta[m x b M p q q A B(C / D) E])\end{array}$ & This study \\
\hline pHT1BH19 & $\begin{array}{l}\text { pUC19 containing } 3.8 \mathrm{~kb} \text { Bam HI-HindIII } \\
\text { fragment }(m x b M \text { and } p q q A B)\end{array}$ & This study \\
\hline pHT2SacH19 & $\begin{array}{l}\text { pUC19 containing } 1.8 \mathrm{~kb} \text { SaclI-HindIII } \\
\text { fragment }\end{array}$ & This study \\
\hline pHT2SacHKan61 & $\begin{array}{l}\text { pAYC61 containing the } 1.8 \mathrm{~kb} \text { SacII-HindIII } \\
\text { fragment of pHT1BH19 with } \mathrm{Km}^{\mathrm{r}} \text { inserted } \\
\text { into the BglII site in } p q q A\end{array}$ & This study \\
\hline pHT3BH19 & $\begin{array}{l}\text { pUC19 containing } 3.8 \mathrm{~kb} \mathrm{BamHI}-\text { HindIII } \\
\text { fragment deleted for pqqA }\end{array}$ & This study \\
\hline pHT3SacH19 & $\begin{array}{l}\text { pUC19 containing } 1.8 \mathrm{~kb} \text { SacII-HindIII } \\
\text { fragment deleted for pqqA }\end{array}$ & This study \\
\hline pHT1 & $\begin{array}{l}\text { pRK310 containing } 7 \cdot 4 \mathrm{~kb} B a m \mathrm{HI}-P s t \mathrm{I} \\
\text { fragment }[m x b M \text { and } p q q A B(C / D) E]\end{array}$ & This study \\
\hline pHT2 & $\begin{array}{l}\text { pRK310 containing } 5 \cdot 4 \mathrm{~kb} \text { Bam HI-PstI } \\
\text { fragment }[p q q A B(C / D) E]\end{array}$ & This study \\
\hline pHT3 & $\begin{array}{l}\text { pRK310 containing } 5 \cdot 1 \mathrm{~kb} \text { Bam HI-PstI } \\
\text { fragment }(p q q(C / D) E)\end{array}$ & This study \\
\hline pHT3L & $\begin{array}{l}\text { pRK310 containing } 7 \cdot 1 \mathrm{~kb} \text { BamHI-PstI } \\
\text { fragment }[m x b M \text { and } p q q(C / D) E]\end{array}$ & This study \\
\hline pHT4 & $\begin{array}{l}\text { pRK310 containing } 5.3 \mathrm{~kb} \text { Bam HI-PstI } \\
\text { fragment }[\text { pqqB(C/D)E] }\end{array}$ & This study \\
\hline pHT4L & $\begin{array}{l}\text { pRK310 containing } 7 \cdot 3 \mathrm{~kb} \mathrm{BamHI}-P s t \mathrm{I} \\
\text { fragment }[m x b M \text { and } p q q B(C / D) E]\end{array}$ & This study \\
\hline pHT2Ns & $\begin{array}{l}\text { pRK310 containing } 0.5 \mathrm{~kb} \text { Bam HI-Pst } \mathrm{I} \\
\text { fragment (pqqA) }\end{array}$ & This study \\
\hline pHT2SacH & $\begin{array}{l}\text { pRK310 containing } 1.8 \mathrm{~kb} \text { Bam HI-HindIII } \\
\text { fragment }(p q q A B)\end{array}$ & This study \\
\hline pHT4SacH & $\begin{array}{l}\text { pRK310 containing } 1.7 \mathrm{~kb} \text { Bam HI-HindIII } \\
\text { fragment }(p q q B)\end{array}$ & This study \\
\hline
\end{tabular}

(Toyama et al., 1997). Transcriptional analysis of $p q q A$ in $M$. extorquens AM1 showed that a short mRNA containing only $p q q A$ was produced at a much higher level than the mRNA for $p q q A B$ (Ramamoorthi \&
Lidstrom, 1995). Similar results were obtained in the case of K. pneumoniae by using pqq-Tn5lacZ operon fusions on the chromosome (Velterop et al., 1995). These results are also consistent with the hypothesis that 
PqqA is a PQQ precursor, because stoichiometric amounts rather than catalytic amounts are required if the peptide is the substrate for PQQ synthesis.

As a first step to examine whether the peptide is the precursor and to elucidate the biochemical steps of PQQ biosynthesis, a deletion mutant of $M$. extorquens AM1 lacking $p q q A B(C / D) E$ was constructed. This mutant was then complemented by a plasmid that contained all of the deleted genes except $p q q A$. Surprisingly, the transformed mutant showed wild-type growth on methanol and significant $P Q Q$ production, suggesting that in $M$. extorquens AM1, $p q q A$ is not required for PQQ production.

\section{METHODS}

Bacterial strains and plasmids. These are listed in Table 1. Genes contained in key plasmids are illustrated in Fig. 1.

Media and growth conditions. M. extorquens AM1 rif and its mutants were grown on the minimal medium described previously (Morris et al., 1994), containing $0.5 \%(\mathrm{v} / \mathrm{v})$ methanol, $0.2 \%(\mathrm{w} / \mathrm{v})$ methylamine hydrochloride, or $0.46 \%$ $(\mathrm{w} / \mathrm{v})$ succinic acid disodium salt hexahydrate. When required, PQQ (Fluka) was added to $1 \mu \mathrm{M}$. E. coli was grown on LB medium. Antibiotics were used as following amounts ( $\mu \mathrm{g}$ $\mathrm{ml}^{-1}$ ): rifamycin, 20; kanamycin, 25 ; tetracycline, 10 ; ampicillin, 50. When production of PQQ was measured, M. extorquens AM1 cells were pre-cultured in $10 \mathrm{ml}$ minimal medium with succinate and appropriate antibiotics, and cultured in
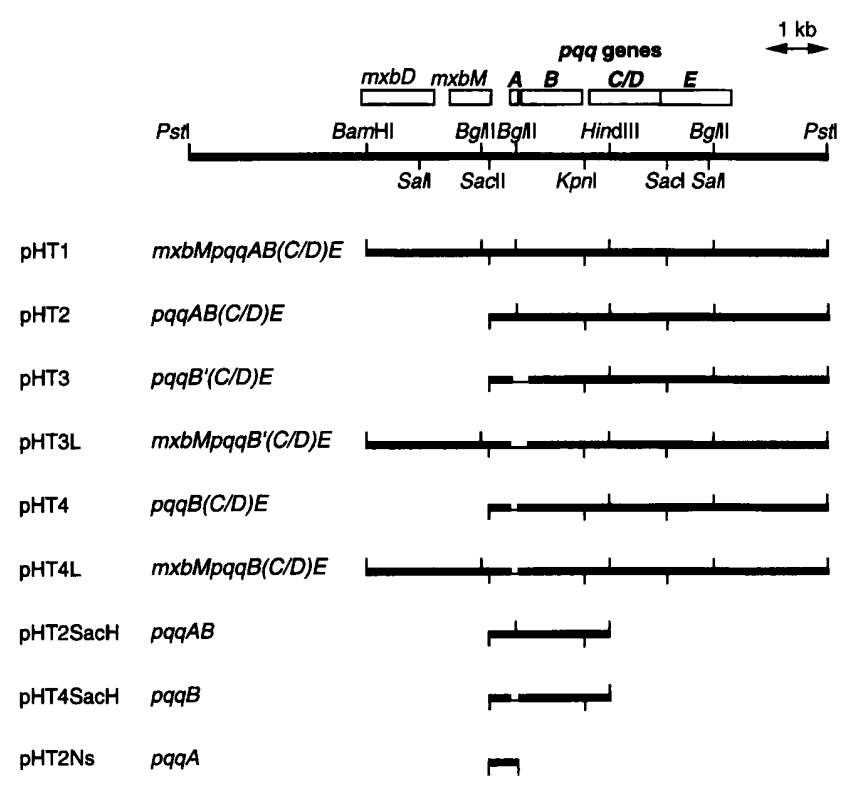

Fig. 1. Schematic representation of plasmids used for complementation experiments. Thick lines show chromosomal DNA regions in each plasmid and thin lines are the deleted region. Open boxes at the top of the figure show the location of $m \times b D M$ and $p q q$ genes; these genes are transcribed from left to right. Details of the construction of plasmids are given in Methods. Note that the Sall and Sacll sites indicated are not unique in this Pstl fragment. GenBank accession numbers are L43136 for the $4.4 \mathrm{~kb}$ Pstl-Bg/ll fragment, L25889 for the $2.0 \mathrm{~kb}$ Bg/ll-HindIII fragment, and U72662 for the $3.6 \mathrm{~kb}$ HindIII-PstI fragment.
$100 \mathrm{ml}$ minimal medium containing appropriate carbon source without antibiotics.

DNA manipulations. Plasmid DNA from E. coli strains was prepared as described by Sambrook et al. (1989). Restriction enzyme digestion, DNA ligation and other DNA modifications were performed according to vendors' recommendations. Oligonucleotides were synthesized at the Biopolymer Synthesis \& Analysis Resource Center at California Institute of Technology. Sequencing reactions and analyses were performed at the Sequencing Core Facility, California Institute of Technology, on an ABI 373A automated sequencer (Applied Biosystems).

Construction of plasmids for mutation. pALS519b and pALS519f, pUC19 derivatives which have a $10 \mathrm{~kb}$ PstI insert containing $m x b M$ and $p q q A B(C / D) E$ and whose orientation of the fragment is opposite to each other, were digested with $B g l I I$ and ligated with the kanamycin-resistance gene cassette digested from pUC4K (Pharmacia) with BamHI. The plasmids obtained were digested with $P v u I I$ and $X b a I$ and then ligated into the same restriction sites of the suicide vector pAYC61 (Chistoserdov et al., 1994). The resultant plasmids were named pALS5Kan61b and pALS5Kan61f.

A pUC19 derivative, pHT1BH19, containing a $3.8 \mathrm{~kb}$ Bam HIHindIII insert (see Fig. 1) was digested with PstI and SacII, treated with T4 DNA polymerase to make blunt ends, and then self-ligated. The resulting plasmid, pHT2SacH19, contains a $1.8 \mathrm{~kb}$ SacII-HindIII fragment. pHT2SacH19 was digested with BglII and ligated with the BamHI-digested kanamycin-resistance gene. The plasmid obtained was digested with PvuII and $X b a I$ and then ligated into the same restriction sites of pAYC61. The resultant plasmid was named pHT2SacHKan61. The construct was confirmed by restriction digest mapping and by sequencing across the ligation junctions.

Construction of plasmids for complementation. pHT1 was constructed with a $7.4 \mathrm{~kb}$ Bam HI-PstI fragment from pALS519b, containing $m x b M$ and $p q q A B(C / D) E$. pHT2, which has the $p q q$ gene cluster but not $m x b M$, was constructed as follows. The $1.8 \mathrm{~kb}$ SacII-HindIII fragment from pHT2SacH19 was isolated as a BamHI-HindIII fragment and ligated into Bam HI/HindIII-digested pUC19 containing the $3.6 \mathrm{~kb}$ HindIII-PstI fragment. The resultant $5.4 \mathrm{~kb} \mathrm{BamHI-}$ PstI fragment was isolated and ligated into the same restriction sites of pRK310, a broad-host-range vector (Ditta et al., 1985).

pHT3L was constructed as follows (see Fig. 2). pHT1BH19, a pUC19 derivative containing the $3.8 \mathrm{~kb}$ BamHI-HindIII fragment, was digested with $P f \mathrm{MI}$ and NsiI, which cut within $p q q A$ and $p q q B$, respectively, removing a $0 \cdot 1 \mathrm{~kb}$ PfiMI-NsiI fragment and an adjacent $0 \cdot 2 \mathrm{~kb} N$ siI fragment. pHT3BH19 was then made by ligating the synthetic oligonucleotides shown in Fig. 2 to the PfiMI/NsiI-digested pHT1BH19. This eliminated all but the first dipeptide of $p q q A$ and produced a deletion of 69 amino acids of $p q q B$. pHT3BH19 was digested with Bam HI and HindIII, and the $3.5 \mathrm{~kb}$ fragment containing $m x b M p q q B^{\prime} C^{\prime}$ was ligated into the HindIII site of pUC19 with the $3.6 \mathrm{~kb}$ HindIII-PstI fragment containing $p q q C / D E$. The resulting $7 \cdot 1 \mathrm{~kb}$ Bam HI-Pst fragment shown in Fig. 2 was isolated and ligated into the same restriction sites of pRK310. The resultant plasmid (pHT3L) contains $m x b M$, $p q q(C / D) E$, and a partial $p q q B$, which would produce $\mathrm{PqqB}$ with a 69 amino acid deletion in the $\mathrm{N}$-terminus, immediately following the first two amino acids (Met His). pHT4L containing $m x b M$ and $p q q B(C / D) E$ was constructed as follows (see Fig. 2). pHT3BH19 was digested with NsiI and the $0.2 \mathrm{~kb}$ NsiI fragment from the $3.8 \mathrm{~kb}$ BamHI-HindIII 


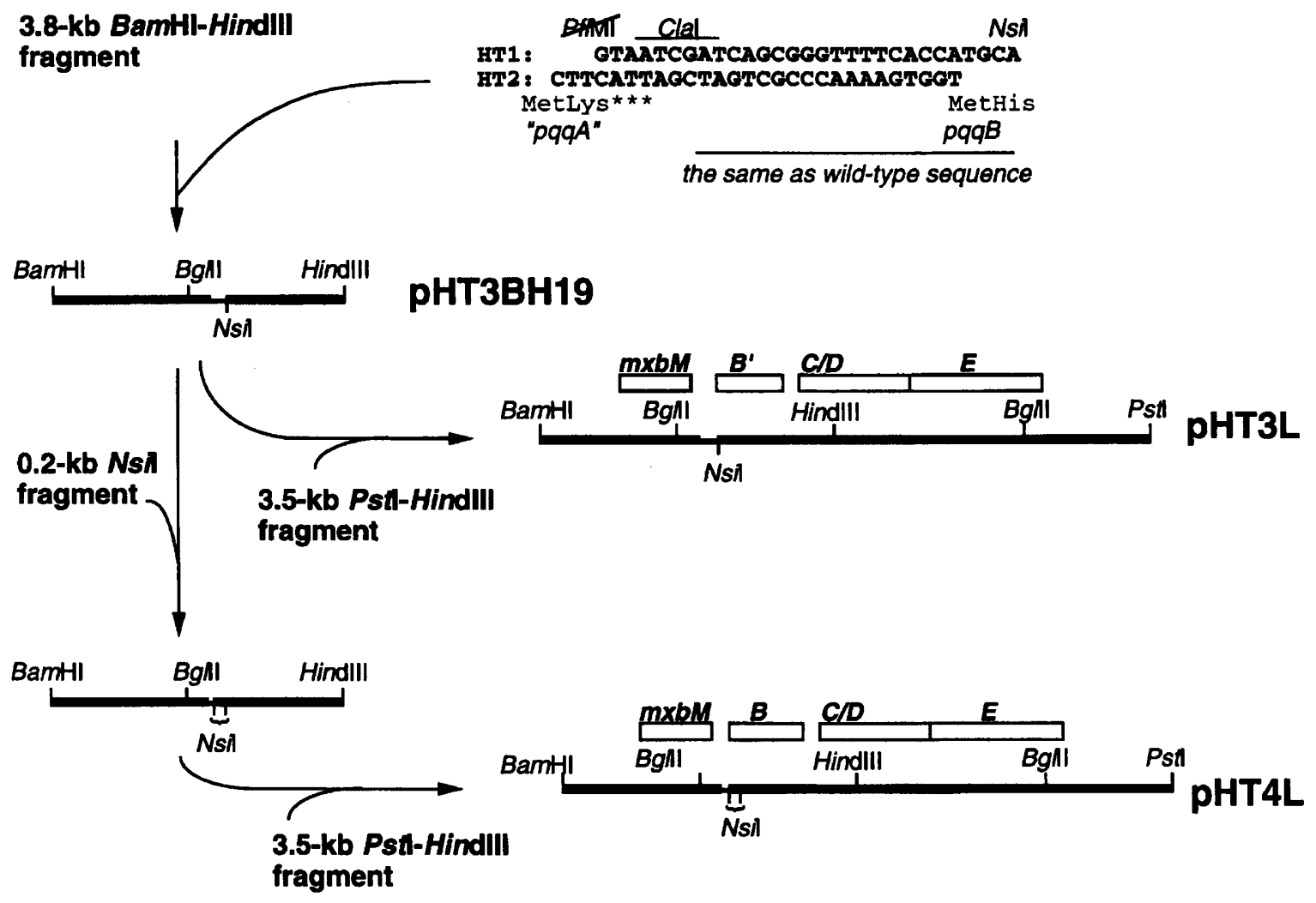

Fig. 2. Schematic representation of the construction of pHT3L and pHT4L. The vector part of each plasmid is not shown. The synthetic oligonucleotides HT1 and HT2 were annealed and inserted into PfiMI/Nsil double-digested pHT3BH19. Detailed description of construction is given in Methods.

fragment was inserted. This NsiI fragment contains the portion of $p q q B$ deleted in $\mathrm{pHT} 3 \mathrm{BH} 19$. The plasmid with correct orientation of the insert was selected by restriction mapping. The $3.7 \mathrm{~kb}$ Bam HI-HindIII fragment was isolated and ligated with the $3.6 \mathrm{~kb}$ HindIII-PstI fragment, and the resulting $7.3 \mathrm{~kb}$ Bam $\mathrm{HI}-P s t \mathrm{I}$ fragment was ligated into the same restriction sites of $\mathrm{pRK} 310$.

pHT3 was constructed as follows. A pUC derivative, pHT3SacH19, was constructed like pHT3BH19 as described above, except the starting fragment was the $1.8 \mathrm{~kb}$ SacIIHindIII fragment. The SacII-HindIII fragment containing the $0.3 \mathrm{~kb}$ deletion was isolated as a Bam HI-HindIII fragment and ligated with the $3.6 \mathrm{~kb}$ HindIII-Pst fragment into pUC19. The $5 \cdot 1 \mathrm{~kb} \mathrm{BamHI-Pst}$ fragment was isolated and ligated into the same restriction sites of pRK310. pHT4 was made by taking advantage of the engineered Clal site in the oligonucleotide insertion in pHT4L (Fig. 2) and pHT3SacH19. The BamHI-ClaI fragment from pHT3SacH19 was combined with the ClaI-HindIII from pHT4L, to generate a $1.7 \mathrm{~kb} \mathrm{BamHI-}$ $H$ indIII fragment with a deleted $p q q A$, and this was combined with the $3.6 \mathrm{~kb}$ HindIII-PstI fragment from pALS519b to generate the $5.3 \mathrm{~kb}$ insert containing $p q q B(C / D) E$. In the case of isolation of the fragments with ClaI cohesive ends, plasmids isolated from E. coli GM2163 (dam $\mathrm{dcm}$ ) were used.

The constructs were confirmed by restriction mapping and by DNA sequencing at the sites of deletion and ligation.

Mating. Plasmids of both pAYC61 and pRK310 derivatives were transferred to $M$. extorquens AM1 rif by using $E$. coli S17-1 as a donor host (Simon et al., 1983). Mating was performed on nitrocellulose filters on nutrient agar (Difco) for $20-24 \mathrm{~h}$ at $30^{\circ} \mathrm{C}$. Transconjugants were selected by rifamycin and kanamycin (for recombinant mutants) or tetracycline (for pRK310 derivatives) resistance on minimal medium containing succinate.

Determination of PQQ content. $M$. extorquens AM1 cells were grown on minimal medium for $48 \mathrm{~h}$. Cells and culture supernatant were separated by centrifugation at $4000 \mathrm{~g}$ for $5 \mathrm{~min}$ at $4^{\circ} \mathrm{C}$. Cells were washed and suspended in $50 \mathrm{mM}$ potassium phosphate buffer ( $\mathrm{pH} 7 \cdot 0$ ) and crude extract was prepared as described previously (Toyama et al., 1997). Crude extracts were pretreated at $100^{\circ} \mathrm{C}$ for $5 \mathrm{~min}$ to release PQQ bound by $\mathrm{MDH}$, prior to PQQ measurements.

The content of PQQ was determined spectrophotometrically as described previously (Toyama et al., 1997), with the apoform of soluble glucose dehydrogenase purified from the $E$. coli cells containing the cloned gene from Acinetobacter calcoaceticus (Cleton-Jansen et al., 1989).

Hybridization with pqqA probe. The $p q q A$ probe (Fig. 3) was tailed with fluorescein-11-dUTP, catalysed by terminal deoxynucleotidyl transferase (Amersham). Chromosomal DNA was isolated by the method of Marmur (1961). Chromosomal DNA $(10 \mu \mathrm{g})$ digested with restriction enzyme(s) was separated by agarose gel electrophoresis, blotted onto a nylon membrane, hybridized with the probe, and detected using the ECL detection system (Amersham) with anti-fluorescein horseradish-peroxidase conjugate. Hybridization temperature was $58^{\circ} \mathrm{C}$ and washing was done at $50^{\circ} \mathrm{C}$ with $1 \times$ SSC containing $0 \cdot 1 \%$ SDS. 


\section{RESULTS}

\section{Construction of a pqqAB(CDD)E deletion mutant}

To study the phenotype of strains containing specific combinations of $p q q$ genes, a mutant was constructed in which the $p q q A B(C / D) E$ gene cluster was deleted. Plasmids were generated (pALS5Kan61b or pALS5Kan61f) in which the $4.1 \mathrm{~kb} B g l \mathrm{lI}$ fragment containing $p q q A B(C / D) E$ and part of $m x b M$ (Fig. 1) had been removed and replaced with a kanamycinresistance gene. No convenient restriction enzyme sites were available between $m x b M$ and $p q q A$, and so this construction was used. $m x b M$ is a gene required for transcription of several methanol oxidation genes, including the MDH subunits (Xu et al., 1993; Springer et al., 1995) and is suggested to be a response-regulator gene of a two-component regulatory system involving $m x b D$, a sensor kinase gene (Springer et al., 1997). It is also involved in transcription of $p q q A$ (Ramamoorthi $\&$ Lidstrom, 1995). pALS5Kan61b or pALS5Kan61f was introduced into E. coli S17-1 ( $m o b$-positive), transferred into $M$. extorquens AM1 rif, and transconjugants were selected on kanamycin and rifamycin and screened on tetracycline. One putative double-crossover insertion mutant (kanamycin-resistant, tetracycline-sensitive) was obtained with pALS5Kan61b and two were obtained with pALS5Kan61f. These three mutants were indistinguishable in carbon source utilization and complementation pattern with plasmids; therefore, only the results with one of them, $3 \mathrm{~b}$, are described hereafter.

\section{Growth of the mutant and its transformants}

Since $m x b M$ is required for expression of the subunits of MDH (Xu et al., 1993; Springer et al., 1995), mutant 3b (with a defective $m x b M$ ) was unable to grow on methanol even in the presence of PQQ. A series of plasmids containing different combinations of $m x b M$ and $p q q$ genes were constructed, and these were tested for complementation of mutant $3 b$ (Fig. 1, Table 2). Wild-type growth of the mutant on methanol was restored with $\mathrm{pHT} 1$, containing $p q q A B(C / D) E$ and $m x b M$. No growth was observed with plasmids that were missing $m x b M$ (pHT2, pHT3, pHT4, pHT2Ns). Surprisingly, wild-type growth was observed by a strain with a plasmid that contained $m x b M$ and $p q q B(C / D) E$, but not $p q q A$. The wild-type growth rate of this strain was confirmed in liquid culture. $\mathrm{pHT} 4$ was shown to contain functional $p q q B, p q q C$ and $p q q E$ genes by complementation of the appropriate $p q q$ mutants (Table 2 ). These results indicate that the $p q q A$ deletion did not cause a polar effect on downstream $p q q$ genes. A plasmid (pHT3L) that contained $m x b M$ and $p q q(C / D) E$ with an in-frame $\mathrm{N}$-terminal truncated form of $p q q B$ allowed slow growth on methanol on agar plates but no growth in liquid culture. However, a similar response was observed with a $p q q B$ mutant containing only the vector, suggesting that PqqB is not absolutely required for growth on methanol in $M$. extorquens AM1. Pqq has been suggested to be involved in the transport of PQQ or its intermediate from the cytoplasm to the periplasm (Velterop et al., 1995).

\section{PQQ production of the wild-type, the mutant and its transformants}

PQQ production was examined in the wild-type, to determine the optimal assay conditions for assessing PQQ production in the mutants. The total PQQ produced by M. extorquens AM1 was measured in both crude extract and culture supernatant, and the majority $(>95 \%)$ was found in the culture supernatant. The

Table 2. Growth on methanol medium of pqq mutants containing different plasmids

Growth was tested on minimal agar plates containing $0.5 \%$ methanol. - , No growth for $3 \mathrm{~d}$; + , reduced growth compared to wild-type cells; ++ , wild-type growth; NT, not tested.

\begin{tabular}{|c|c|c|c|c|c|}
\hline \multirow[t]{2}{*}{ Plasmid } & \multirow[t]{2}{*}{ Gene(s) } & \multicolumn{4}{|c|}{ Growth of mutants: } \\
\hline & & $\begin{array}{c}3 \mathrm{~b} \\
{[\Delta p q q A B(C / D) E} \\
m x b M]\end{array}$ & $\begin{array}{c}\text { UV45 } \\
(p q q E)\end{array}$ & $\begin{array}{c}\text { EMS12 } \\
(p q q C / D)\end{array}$ & $\begin{array}{c}\text { UV46 } \\
\text { (pqqB) }\end{array}$ \\
\hline pRK310 & None & - & - & - & $-*$ \\
\hline pHT1 & $m x b M p q q A B(C / D) E$ & ++ & NT & NT & NT \\
\hline pHT2 & $p q q A B(C / D) E$ & - & ++ & ++ & ++ \\
\hline pHT3 & $p q q B^{\prime}(C / D) E$ & - & ++ & ++ & $-*$ \\
\hline pHT3L & $m x b M p q q B^{\prime}(C / D) E$ & + & NT & NT & NT \\
\hline pHT4 & $p q q B(C / D) E$ & - & ++ & ++ & ++ \\
\hline pHT4L & $m x b M p q q B(C / D) E$ & ++ & NT & NT & NT \\
\hline pHT2Ns & $p q q A$ & - & - & NT & $-*$ \\
\hline pHT2SacH & $p q q A B$ & NT & NT & NT & ++ \\
\hline pHT4SacH & $p q q B$ & NT & NT & NT & ++ \\
\hline
\end{tabular}

* No growth for $3 \mathrm{~d}$, but significant growth was observed after 1 week. 

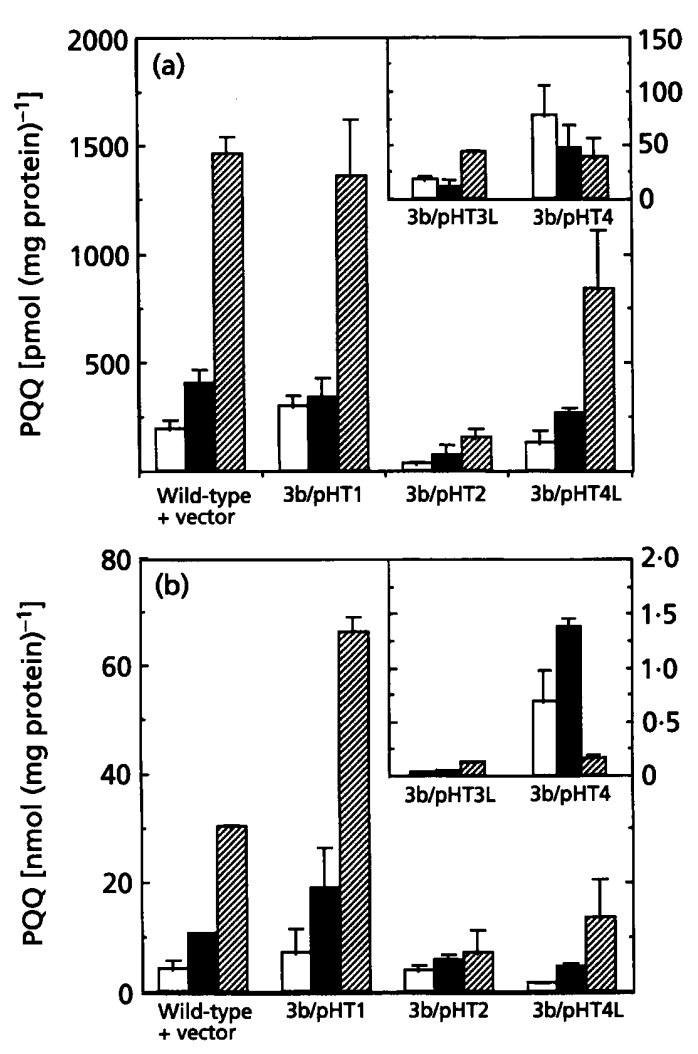

Fig. 3. $P Q Q$ amounts produced in the transformants: (a) in crude extract; (b) in total of crude extract and culture supernatant. PQQ amount was measured as described in Methods. Results are expressed as amounts per $\mathrm{mg}$ protein in the crude extract, to normalize all values to the amount of cells present. Open, black and hatched bars are the results of cultures grown with succinate, methanol plus methylamine, and methanol, repectively. Error bars show standard deviations of two to four experiments.

total PQQ produced was usually around $30 \mathrm{nmol}(\mathrm{mg}$ protein) ${ }^{-1}$, which corresponded to about $10 \mu \mathrm{M}$ in the culture supernatant. When PQQ production was followed over time, it was found that PQQ appeared in the culture supernatant only after substantial growth had occurred. The amount of PQQ continued to increase as the culture entered stationary phase and then it plateaued. Therefore, PQQ production of the mutant and its transformants was measured in early stationary phase. Mutants and transformants that did not show growth on methanol were tested for PQQ production after $48 \mathrm{~h}$ incubation in the presence of methanol.

The amount of PQQ produced by the mutants was checked both in the culture supernatant and the crude extract in cells grown under three conditions, with succinate (non-inducing), with methanol (inducing, but no growth for some of the mutants), and with methanol plus methylamine (inducing, all strains grow) (Fig. 3). Crude extract was heat-treated to release the PQQ bound by MDH prior to assaying PQQ. In the wildtype, PQQ was detectable in crude extract prior to heat treatment, but it was only a trace amount as compared to the amount after heat treatment. PQQ amounts detected in the crude extract were similar to that of wildtype for strains that could not produce $\mathrm{MDH}$ (mutant $3 \mathrm{~b}$ with pHT2 or pHT4), and the amounts detected were similar before and after heat treatment of the crude extract. These results suggest that most of the PQQ in crude extract was bound to MDH in the wild-type. In all cases, PQQ was mainly found in the culture supernatant and only a small percentage of the total PQQ was in the crude extract, except in the case of the mutant $3 \mathrm{~b}$ with pHT3L. This plasmid lacks $p q q A B$, and in this strain over half of the small amount of PQQ produced was found in the crude extract. This result supports the results observed in $K$. pneumoniae, suggesting that $\mathrm{PqqB}$ is involved in the transport of PQQ (Velterop et al., 1995).

Maximum production of PQQ by the wild-type or its transformant with the vector pRK310 was observed when they grew on methanol, and about one-seventh that amount was produced by cells grown on succinate (Fig. 3). Intermediate levels were observed in cells grown on methanol plus methylamine. Neither the crude extract nor the culture supernatant of the mutant $3 \mathrm{~b}$ with pRK310 contained detectable PQQ when grown under any of the three conditions (data not shown). The mutant with pHT1 produced PQQ in larger amounts than the wild-type under all three conditions, presumably as a result of the higher copy number of $p q q$ genes. When the plasmid pHT2 (no $m x b M$ ) was introduced, succinate-grown cells produced about the same amount of PQQ as wild-type, but this amount did not increase significantly under inducing conditions. These results suggest that $m x b M$ is essential for normal induction of PQQ production by methanol. The transformant with pHT4L (missing only pqqA) was able to grow on methanol and produced PQQ at levels about one-half to one-third that of wild-type cells, and onefifth to one-tenth that of the deletion mutant containing pHT1. The PQQ production pattern in this strain was similar to the wild-type. The transformant containing pHT4 (no $m x b M$ or $p q q A$ ) showed lower levels of PQQ as compared to the transformant containing pHT2 (no $m x b M$ ), and in keeping with the proposed role of $m x b M$ in PQQ induction, this strain did not show methanol induction. In fact, the levels on methanol were lower than in the other two growth conditions. The mutant harbouring $\mathrm{pHT} 3 \mathrm{~L}$ (missing $p q q A B$ ) produced low but detectable amounts of PQQ in all conditions, and showed increased production of PQQ by cells transferred into medium containing methanol, although no growth was observed. No PQQ production was detected in the transformant with pHT3 (no $m x b M$ or $p q q A B$ ), presumably reflecting accumulative defects in $p q q A B$ and loss of induction by $m x b M$.

\section{Construction of a pqqA insertion mutant and its properties}

The data obtained with the large deletion mutant suggested that $p q q A$ was not essential for PQQ production in M. extorquens AM1. To examine the role of the small peptide further, another mutant was isolated 
M. extorquens AM1: GTC GGC ATG GAA GTC ACG AGC TAC GAG

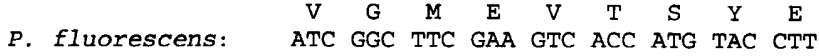

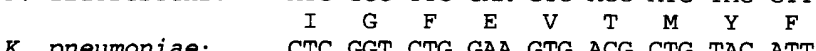
k. pneumoniae: CTC GGT CTG GAA GTG ACG CTG TAC ATT A. calcoaceticus: ATT GGT TTT GAA GTT ACA ATG TAC TTT $\begin{aligned} & \text { Probe: GGC ATG GAA GTC ACG AGC TAC } \\ & \text { T C } \text { G } \text { C CTG }\end{aligned}$

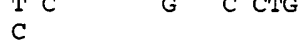

Fig. 4. The conserved region of known pqqA genes, with the probe used for detection of pqqA-like genes shown below.

by homologous recombination with pHT2SacHKan61, which has a kanamycin-resistance gene inserted in the $B g l$ II site in $p q q A$, between the start codon and the conserved region of PqqA shown in Fig. 4. Two putative double-crossover recombinants were isolated (kanamycin-resistant, tetracycline-sensitive). The doublecrossover event was analysed for both recombinants by probing diagnostic digests of chromosomal DNA using an oligonucleotide probe targeted to the region immediately downstream of the insertion. The restriction enzymes used were chosen so that they cut within the kanamycin-resistance gene and downstream of the probed sequence. The hybridization revealed in each case that the wild-type bands had shifted to the predicted size, confirming the insertion event at the correct site within $p q q A$. These mutants were indistinguishable and one of them, D2, was used for further experiments. The mutant D2 grew on agar plates containing methanol as well as the wild-type cells, and the maximum growth rate in liquid culture was similar to that of the wild-type cells grown on succinate or methanol. However, total PQQ production was only $5-11 \%$ of the wild-type in a $48 \mathrm{~h}$ culture (Table 3 ). Most of this decrease was in the culture supernatant fraction, as the PQQ in the crude extract was still about half that found for the wild-type cells. A time course of PQQ production by methanolgrown D2 cells showed that PQQ eventually reached about $4 \mu \mathrm{M}$ in the culture supernatant when cells were

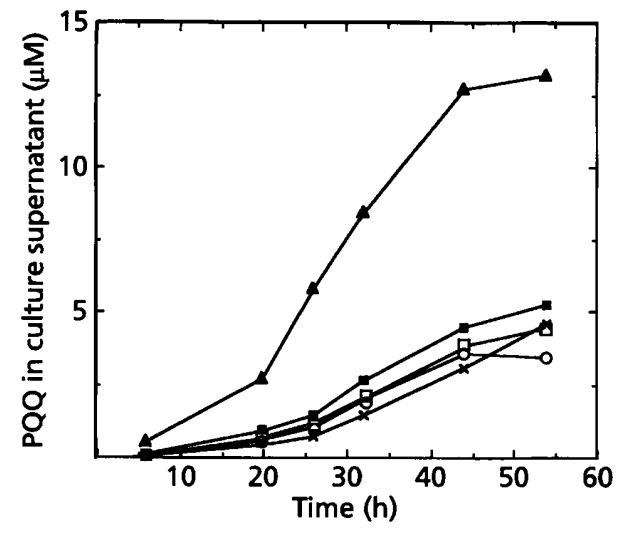

Fig. 5. $P Q Q$ production of the mutant $D 2$ and its transformants. Cells were grown on succinate and transferred to the same volume of fresh medium containing methanol at time zero. The PQQ amount in each culture supernatant was measured as described in Methods. Symbols represent transformants of the mutant D2 with pRK310 (O), pHT2Ns $(\times)$, pHT2SacH $(\square)$, pHT2 (A) and pHT4 (U).

incubated longer after entering stationary phase (Fig. 5). This stationary-phase culture was diluted and spread on agar medium with either methanol alone or succinate plus kanamycin. The cell numbers on both agar plates were similar, indicating that no significant levels of kanamycin-sensitive revertant cells were present in the culture.

Plasmids containing $p q q A$ were transferred into the $\mathrm{D} 2$ mutant, and PQQ produced in the culture medium was measured (Fig. 5). Both pHT2Ns, which contains only $p q q A$ and $\mathrm{pHT} 2 \mathrm{SacH}$, which contains $p q q A B$, had little effect on PQQ production. pHT2 increased PQQ production to that of the wild-type cells or more (Fig. 5). Failure to complement the $p q q A$ mutant with pHT2Ns and $\mathrm{pHT} 2 \mathrm{SacH}$ might be explained by polarity from the kanamycin-resistance gene insertion. However, pHT4, which contains $p q q B(C / D) E$ but not $p q q A$, increased PQQ production only slightly.

Table 3. PQQ produced into the culture supernatant by the mutant D2 grown on different substrates

PQQ amounts were measured as described in Methods, and normalized to the amount of protein in the crude extract.

\begin{tabular}{|c|c|c|c|}
\hline & \multicolumn{3}{|c|}{ Amount of PQQ [nmol (mg protein $\left.)^{-1}\right]$ in cells grown on: } \\
\hline & Succinate & $\begin{array}{l}\text { Methanol + } \\
\text { methylamine }\end{array}$ & Methanol \\
\hline \multirow{2}{*}{ In culture supernatant } & $(97 \cdot 1 \pm 31 \cdot 5)^{*}$ & $(364 \pm 245)^{*}$ & $(610 \pm 450)^{*}$ \\
\hline & $0.441 \pm 0.17$ & $1 \cdot 42 \pm 1 \cdot 2$ & $1 \cdot 81 \pm 1 \cdot 24$ \\
\hline In crude extract & $0 \cdot 18 \pm 0.05$ & $0.29 \pm 0.09$ & $0.69 \pm 0.26$ \\
\hline Total amount produced & $0.59 \pm 0.12$ & $1 \cdot 71 \pm 1 \cdot 11$ & $2 \cdot 50 \pm 0.98$ \\
\hline
\end{tabular}

*Values in parentheses are $\mathrm{nM}$. 


\section{Detection of genes similar to pqqA}

It is possible that a second $p q q A$ or a different gene that produces a peptide similar to PqqA exists in $M$. extorquens AM1. If so, this would explain why the $p q q A$ mutants are able to produce sufficient amounts of PQQ for normal growth on methanol. Hybridization experiments were carried out to search for $p q q A$-like genes in the DNA of $M$. extorquens AM1. The probe was designed to take into account the conserved amino acids present in the known PqqA peptides and the codon bias of M. extorquens AM1 (Fig. 4). This probe showed only one signal, a $4.5 \mathrm{~kb}$ Sali fragment or a $3.8 \mathrm{~kb}$ Bam HI-HindIII fragment in digested genomic DNA from wild-type cells, the fragments known to contain pqqA. No signals were detected in the digested genomic DNA of the mutant $3 b$ or for the plasmid pHT4L, confirming the lack of $p q q A$ or a $p q q A$-like gene in these cases. Further experiments at lower stringencies also failed to identify a second band with the $p q q A$ probe.

\section{DISCUSSION}

A mutant of $M$. extorquens AM1 lacking the $p q q$ gene cluster $p q q A B(C / D) E$ was obtained and analysed by complementation. Unexpectedly, strains complemented with a plasmid containing $p q q B(C / D) E$ but not $p q q A$ grew well on methanol, with a PQQ production of $10-20 \%$ that of the wild-type containing the entire $p q q$ gene cluster on a plasmid. This phenotype was confirmed with another mutant, in which a kanamycinresistance gene was inserted within pqqA in the chromosome. This mutant also grew well on methanol, even though its PQQ production was about $10-20 \%$ that of the wild-type. However, the crude extract of this mutant contained PQQ amounts about half that of the wildtype. Since the majority of the PQQ in crude extracts appears to be found in $\mathrm{MDH}$, these data suggest that the reduced production of PQQ in the mutant was still sufficient to generate enough active $\mathrm{MDH}$ to support normal growth of the mutant on methanol. Therefore, disruption of $p q q A$ reduced production of PQQ, but did not abolish it.

These results are surprising, because several lines of evidence suggest that the small peptide encoded by $p q q A$ is directly involved in the formation of PQQ. In $A$. calcoaceticus, site-directed mutagenesis at the conserved tyrosine or glutamate residue abolished the production of PQQ (Goosen et al., 1992), and a frame-shift mutation in $p q q A$ of $K$. pneumoniae also abolished PQQ synthesis (Meulenberg et al., 1992). The latter mutation could be complemented in trans (Velterop et al., 1995), suggesting that the translated peptide itself might be involved in PQQ synthesis. PqqF of $M$. extorquens AM1 and $K$. pneumoniae show identity to the mitochondrial processing peptidase family and PqqG of $M$. extorquens AM1 shows identity to the pitrilysin/insulin-degrading enzyme family (Springer et al., 1996), implying that they could cleave the peptide or its derivative in the course of PQQ synthesis. In addition, the steady-state level of $p q q A$ mRNA is very high compared to mRNA for the rest of the gene cluster, as would be expected for a precursor, and the predicted ribosome-binding site of $p q q A$ is very strong while those of the other $p q q$ genes are weak, which could further enhance the levels of PqqA over the other $p q q$ gene products (Ramamoorthi $\&$ Lidstrom, 1995). All of these data support the hypothesis that the PqqA peptide is the precursor of PQQ. It is possible that a peptide similar to PqqA is present in M. extorquens AM1 that can take the place of PqqA in PQQ synthesis, but an attempt to find such a gene failed with a probe based on conserved pqqA DNA sequences available from four bacteria. We cannot exclude the possibility that a peptide encoded by a gene that was not detected in the hybridization experiment is used for PQQ synthesis in the pqqA mutants. Alternatively, it is possible that something other than the peptide might be involved in PQQ synthesis, either as the normal synthesis pathway or as a less efficient route only revealed in the PqqA mutant. For instance, it is possible that in $M$. extorquens AM1 free tyrosine and glutamate might generate PQQ with lower efficiency than PqqA. Whatever this alternative pathway might be, it requires all of the other $p q q$ genes. Our results suggest that an in vitro system for producing PQQ using a labelled peptide will be necessary to determine whether the peptide can be the precursor of PQQ.

Our results also demonstrate that $m x b M$ is required for normal methanol induction of PQQ synthesis. Previous results had shown that $\mathrm{MxbM}$ has similarity to a family of response-regulator proteins, and together with $\mathrm{MxbD}$, apparently forms a sensor-regulator pair involved in transcription of a number of methanol oxidation genes including $p q q A$ (Ramamoorthi et al., 1995; Springer et al., 1997). We have now shown that even in strains lacking $p q q A$, the production of PQQ shows the normal regulatory pattern if $m x b M$ is present, but not if this gene is absent. These results show that whatever the route of PQQ synthesis in the $p q q \mathrm{~A}$ mutants may be, it is methanol-inducible and this regulation is mediated by the $m x b D M$ system.

\section{ACKNOWLEDGEMENTS}

This research was supported by a grant from the NIH (GM36294). H. T. was supported by Postdoctoral Fellowships for Research Abroad from the Japan Society of the Promotion of Science.

\section{REFERENCES}

Anthony, C., Ghosh, M. \& Blake, C. C. F. (1994). The structure and function of methanol dehydrogenase and related quinoproteins containing pyrrolo-quinoline quinone. Biochem J 304, 665-674.

Chistoserdov, A. Y., Chistoserdova, L. V., Mclntire, W. S. \& Lidstrom, M. E. (1994). Genetic organization of the mau gene cluster in Methylobacterium extorquens AM1: complete nucleotide sequence and generation and characterization of mau mutants. J Bacteriol 176, 4052-4065.

Cleton-Jansen, A.-M., Goosen, N., Vink, K. \& Putte, P. V. D. (1989). Cloning, characterization and DNA sequencing of the gene encoding the $M_{\mathrm{r}} 50,000$ quinoprotein glucose dehydrogenase from Acinetobacter calcoaceticus. Mol Gen Genet 217, 430-436. 
Ditta, G., Schmidhauser, T., Yakobson, E., Lu, P., Liang, X.-W., Finlay, D. R., Guiney, D. \& Helinski, D. R. (1985). Plasmids related to the broad host range vector pRK290, useful for gene cloning and for monitoring gene expression. Plasmid 13, 149-153.

Gomelsky, M., Biville, F., Gasser, F. \& Tsygankov, Y. D. (1996). Identification and characterization of the $p q q D G C$ gene cluster involved in pyrroloquinoline quinone production in an obligate methylotroph Methylobacillus flagellatum. FEMS Microbiol Lett 141, 169-176.

Goosen, N., Horsman, H. P. A., Huinen, R. G. M. \& Putte, P. V. D. (1989). Acinetobacter calcoaceticus genes involved in biosynthesis of the coenzyme pyrrolo-quinoline-quinone: nucleotide sequence and expression in Escherichia coli K-12. J Bacteriol 171, 447-455.

Goosen, N., Huinen, R. G. M. \& Putte, P. V. D. (1992). A 24-aminoacid polypeptide is essential for the biosynthesis of the coenzyme pyrrolo-quinoline-quinone. J Bacteriol 174, 1426-1427.

Houck, D. R., Hanners, J. L. \& Unkefer, C. J. (1991). Biosynthesis of pyrroloquinoline quinone. 2. Biosynthetic assembly from glutamate and tyrosine. J Am Chem Soc 113, 3162-3166.

Kleef, M. A. G. v. \& Duine, J. A. (1988). L-Tyrosine is the precursor of PQQ biosynthesis in Hyphomicrobium X. FEBS Lett 237, 91-97.

Marmur, J. (1961). A procedure for the isolation of deoxyribonucleic acid from micro-organisms. J Mol Biol 3, 208-218.

Matsushita, K. \& Adachi, O. (1993). Bacterial quinoprotein dehydrogenases glucose dehydrogenase and alcohol dehydrogenase. In Principles and Applications of Quinoproteins, pp. 47-64. Edited by V. L. Davidson. New York: Marcel Dekker.

Meulenberg, J. J. M., Sellink, E., Riegman, N. H. \& Postma, P. W. (1992). Nucleotide sequence and structure of the Klebsiella pneumoniae pqq operon. Mol Gen Genet 232, 284-294.

Morris, C. J., Biville, F., Turlin, E., Lee, E., Ellermann, K., Fan, W.-H., Ramamoorthi, R., Springer, A. L. \& Lidstrom, M. E. (1994). Isolation, phenotypic characterization and complementation analysis of mutants in Methylobacterium extorquens AM1 unable to synthesize pyrroloquinoline quinone and sequence of $p q q D$, $p q q G$, and pqqC. J Bacteriol 176, 1746-1755.

Nunn, D. N. \& Lidstrom, M. E. (1986). Isolation and complementation analysis of 10 methanol oxidation mutant classes and identification of the methanol dehydrogenase structural gene of Methylobacterium sp. strain AM1. J Bacteriol 166, 581-590.

Ramamoorthi, R. \& Lidstrom, M. E. (1995). Transcriptional analysis of $p q q D$ and study of the regulation of pyrroloquinoline quinone biosynthesis in Methylobacterium extorquens AM1. J Bacteriol 177, 206-211.

Sambrook, J., Fritsch, E. F. \& Maniatis, T. (1989). Molecular Cloning: a Laboratory Manual, 2nd edn. Cold Spring Harbor, NY: Cold Spring Harbor Laboratory.

Schnider, U., Keel, C., Voisard, C., Defago, G. \& Haas, D. (1995). Tn5-directed cloning of pqq genes from Pseudomonas fluorescens $\mathrm{CHA} 0$ : mutational inactivation of the genes results in overproduction of the antibiotic pyoluteorin. Appl Environ Microbiol 61, 3856-3864.

Simon, R., Priefer, U. \& Puhler, A. (1983). A broad host range mobilization system for in vivo genetic engineering: transposon mutagenesis in Gram negative bacteria. Bio/Technology 1, 784-791.

Springer, A. L., Chou, H.-H., Fan, W.-H., Lee, E. \& Lidstrom, M. E. (1995). Methanol oxidation mutants in Methylobacterium extorquens AM1: identification of new genetic complementation groups. Microbiology 141, 2985-2993.

Springer, A. L., Ramamoorthi, R. \& Lidstrom, M. E. (1996). Characterization and nucleotide sequence of $p q q E$ and $p q q F$ in Methylobacterium extorquens AM1. J Bacteriol 178, $2154-2157$.

Springer, A. L., Morris, C. J. \& Lidstrom, M. E. (1997). Molecular analysis of $m x b D$ and $m x b M$, a putative sensor-regulator pair required for oxidation of methanol in Methylobacterium extorquens AM1. Microbiology 143, 1737-1744.

Toyama, H., Chistoserdova, L. \& Lidstrom, M. E. (1997). Sequence analysis of $p q q$ genes of Methylobacterium extorquens AM1 required for biosynthesis of pyrroloquinoline quinone and purification of a biosynthetic intermediate. Microbiology 143, 595-602.

Velterop, J. S., Sellink, E., Meulenberg, J. J. M., David, S., Bulder, I. \& Postma, P. W. (1995). Synthesis of pyrroloquinoline quinone in vivo and in vitro and detection of an intermediate in the biosynthetic pathway. J Bacteriol 177, 5088-5098.

Yanisch-Perron, C., Vieira, J. \& Messing, J. (1985). Improved M13 phage cloning vectors and host strains : nucleotide sequences of the M13mp18 and pUC19 vectors. Gene 33, 103-119.

Xu, H. H., Viebahn, M. \& Hanson, R. S. (1993). Identification of methanol-regulated promoter sequences from the facultative methylotroph Methylobacterium organophilum XX. J Gen Microbiol 139, 743-752.

Received 19 June 1997; revised 12 September 1997; accepted 19 September 1997. 